\title{
Rigueur budgétaire, crédibilité des politiques budgétaires et comportement de consommation
}

\author{
PATRICK ARTUS*
}

\section{INTRODUCTION}

De nombreux pays ont réduit ou vont réduire dans le futur leurs déficits et leurs dettes publics. Il est important pour ces pays que les politiques budgétaires restrictives qu'ils mettent en place ne freinent pas l'activité, d'autant plus qu'en général ils se trouvent, au moment de mettre en oeuvre ces politiques, déjà dans une situation de chômage keynésien.

La theorie concernant les effets sur l'activité des politiques budgétaires restrictives paraît assez claire. Si les consommateurs sont rationnels, ces politiques n'ont pas d'effet de freinage sur l'activité lorsqu'elles consistent en réduction des dépenses. En effet, il y a alors baisse des impôts futurs anticipés, donc hausse du revenu disponible et de la consommation privée, ce qui compense la réduction de la consommation publique [MILLER-SKIDELVSY-WELLER (1990); FELDSTEIN (1982); BERTOLA-DRAZEN (1993)]. Comme nous le verrons, cette conclusion de départ doit être précisée en fonction des effets des politiques sur la production et le revenu. A l'inverse, des effets keynésiens des déficits publics peuvent apparaître si l'horizon des consommateurs est tel qu'ils pensent que l'ajustement fiscal ne les concernera pas [SUTHERLAND (1995)].

Les analyses empiriques confirment en général ces analyses théoriques. Les effets non keynésiens des politiques budgétaires restrictives ont été observés dans plusieurs pays européens [GIAVAZZI-PAGANO (1990) et (1995)] (nous donnerons quelques indications empiriques plus loin). La réduction du déficit est durable lorsqu'il y a baisse des dépenses et non lorsqu'il y a hausse de la pression fiscale [ALESINA-PEROTTI (1995)].

Il faut aussi se poser la question importante de la crédibilité des autorités. Si par exemple une réduction des dépenses publiques n'est perçue que comme transitoire, elle n'implique qu'une faible stimulation de la consommation privée. ll faut donc normalement qu'elle soit anticipée comme durable pour ne pas freiner l'activité. A l'inverse, une hausse des dépenses publiques a, si les autorités ne sont pas perçues comme recherchant la solvabilité du budget, des effets de stimulation de l'activité, puisque les agents privés ne pensent pas qu'elle conduise nécessairement à une hausse des impôts [ARTUS (1996)].

* Directeur des études économiques de la Caisse des dépôts et consignations

Professeur associé à l'Université Paris I

Professeur à l'école Polytechnique 
On devrait donc examiner la crédibilité (la réputation) des autorités fiscales de la même manière que la réputation des autorités a été examinée par la littérature ${ }^{1} ; \mathrm{y}-\mathrm{a}$-t-il incitation à tricher une fois que des politiques ont été annoncées? Quel est le gain du passage à l'équilibre coopératif entre autorités et secteur privé, c'est-à-dire de la "crédibilité fiscale»? L'annonce de la politique budgétaire future modifie, si elle est crédible, les choix de consommation, donc l'activité économique à court terme.

C'est de cet effet d'annonce que peut provenir le problème de crédibilitét ${ }^{2}$ dans un sens ou dans l'autre: les autorités ont une incitation à annoncer des déficits futurs élevés, si l'équilibre économique est de type keynésien, pour stimuler l'activité (donc accroître les revenus anticipés et la consommation présente); par contre, si l'activité future n'est pas anticipée rationnellement, l'effet dominant est celui des impôts futurs anticipés. Les autorités peuvent alors avoir intérêt à annoncer des déficits futurs réduits pour accroître le revenu (après taxes) anticipé et la consommation.

Il faut aussi analyser les liens qui existent entre la nature des anticipations des consommateurs pour les différentes variables (déficit, production...), «l'incitation à tricher» des autorités, leur horizon temporel et le profil dans le temps des déficits publics.

Nous nous posons la question du passage à des politiques fiscales plus restrictives, que nous représentons comme l'apparition d'une contrainte de stabilisation précoce de la dette publique, et d'une contrainte d'équilibre ${ }^{3}$ budgétaire ultérieure, comme par exemple dans le traité de Maastricht. Au lieu d'être confrontées au besoin d'assurer à long terme la solvabilité budgétaire, les autorités doivent l'assurer rapidement. Nous nous demandons ce que sont les effets et les motivations de ce type de rigueur fiscale, par rapport à la situation antérieure.

Les programmes de réduction rapide des déficits publics observés dans divers pays européens correspondent bien à cette politique de «solubilisation rapide». Dans plusieurs cas, cette réduction rapide n'a pas entraîné de conséquences économiques néfastes, au contraire.

Les tableaux 1 et 2 ci-dessous montrent que dans trois cas (Danemark, Irlande, Suède), la réduction du déficit, total ou structurel, a été extrêmement forte, rapide et de plus durable. Dans ces trois cas, au terme de la période de restriction, le rapport de la dette publique au PIB baisse, ce qui correspond bien à l'apparition d'une contrainte brutale de stabilisation. Dans les trois cas également, on observe que la croissance non seulement n'a pas faibli, mais a accéléré, grâce à une baisse forte du taux d'épargne des ménages, qui stimule la consommation. Le point commun entre ces trois cas est la baisse forte des dépenses publiques, alors que la situation est diverse en ce qui concerne la pression fiscale (baisse dans un cas, hausse dans deux autres).

1. Par exemple, BARRO-GORDON (1983).

2. Comme chez ChARI-KeHOE (1990), la taxe optimale intertemporelle n'est pas cohérente temporellement.

3. On sait que les conflits entre groupes sociaux peuvent provoquer un retard dans les politiques d'ajustement [ALESINA-DRAZEN (1991)]. 
Tableau 1

Réductions de déficit public (\% du PIB)

\begin{tabular}{|l|c|c|c|c|}
\hline & $\begin{array}{c}\text { Variation du } \\
\text { déficit total }\end{array}$ & $\begin{array}{c}\text { Variation du } \\
\text { déficit structurel }\end{array}$ & $\begin{array}{c}\text { Caractère durable } \\
\text { de la réduction } \\
\text { du déficit* }\end{array}$ & $\begin{array}{c}\text { Excédent } \\
\text { primaire final }\end{array}$ \\
\hline $\begin{array}{l}\text { Danemark } \\
1982 \text { à } 1986\end{array}$ & 13 & 9,5 & oui & 8,5 \\
$\begin{array}{l}\text { Irlande } \\
1986 \text { à 1989 } \\
\begin{array}{l}\text { Suède } \\
1985 \text { à } 1987\end{array}\end{array} \quad 8$ & 7,5 & oui & 2 \\
\hline
\end{tabular}

* Réduction maintenue après 3 ans

Tableau 2

Conditions de réductions de déficit

\begin{tabular}{|l|c|c|c|c|}
\hline & $\begin{array}{c}\text { Variation de la } \\
\text { pression fiscale } \\
\text { (\% du PIB) }\end{array}$ & $\begin{array}{c}\text { Variation des } \\
\text { dépenses publiques } \\
\text { (\% du PIB) }\end{array}$ & $\begin{array}{c}\text { Variation de la } \\
\text { croissance du PIB } \\
(\% \text { par an })\end{array}$ & $\begin{array}{c}\text { Variation du taux } \\
\text { d'épargne* } \\
\text { (point) }\end{array}$ \\
\hline $\begin{array}{l}\text { Danemark } \\
1982 \text { à } 1986\end{array}$ & $+7,1$ & $-5,5$ & $+2,9$ & $-2,0$ \\
$\begin{array}{l}\text { Irlande } \\
1986 \text { à } 1989\end{array}$ & $-3,0$ & $-12,1$ & $+1,3$ & $-3,4$ \\
$\begin{array}{l}\text { Suède } \\
1985 \text { à } 1987\end{array}$ & $+2,6$ & $-5,5$ & $+0,7$ & $-5,9$ \\
\hline
\end{tabular}

* Entre la période antérieure et la période de réduction des déficits

Nous nous demanderons dans ce qui suit si on peut interpréter ces caractéristiques de ces trois épisodes de rigueur budgétaire brutale.

Nous commençons donc par examiner la nature des effets de la rigueur fiscale selon le mode de formation des anticipations et le degré de crédibilité des autorités. Nous supposons qu'à court terme l'équilibre est keynésien, et que les autorités peuvent modifier le niveau de production; qu'à long terme, où la contrainte de solvabilité s'exerce, la production est donnée. Nous verrons que le fait que la production varie avec la demande modifie les conclusions les plus élémentaires: une hausse des dépenses publiques d'une part accroît les impôts futurs anticipés (donc freine la consommation), mais d'autre part stimule la production, donc les revenus, ce qui va en sens opposé et n'est pas toujours pris en compte.

Nous examinons ensuite les effets qu'on peut attendre d'une contrainte de «solvabilité précoce» pour les dépenses publiques. Nous verrons aussi que les effets usuellement décrits dépendent d'hypothèses bien spécifiques. Finalement nous nous interrogerons sur la nécessité de règles budgétaires strictes en Europe après l'unification. 


\section{L'EFFET DES POLITIQUES DE RÉDUCTION DES DÉPENSES PUBLIQUES SUR L'ACTIVITÉ ÉCONOMIQUE: LE RÔLE CENTRAL DES ANTICIPATIONS}

La façon dont l'évolution observée dans les petits pays européens est usuellement expliquée est simple: la réduction des dépenses publiques conduit à une révision à la baisse des impôts futurs anticipés, d'où un supplément de dépense privée qui compense la réduction des dépenses publiques. La hausse des dépenses privées est d'autant plus forte que les autorités fiscales sont crédibles, c'est-à-dire que la baisse observée à court terme des dépenses publiques est anticipée devoir être durable.

Quand on réfléchit à cette explication usuelle, on s'aperçoit que les mécanismes peuvent être plus complexes. Décomposons le temps en trois périodes: aujourd'hui, le moyen terme, le long terme. Si les autorités annoncent une réduction des dépenses publiques aujourd'hui et dans un moyen terme, et si l'équilibre économique est encore de type keynésien à moyen terme, il en résulte une baisse de la production (déterminée par la demande) anticipée, donc une baisse de la consommation à court terme. L'effet est donc complètement différent de celui usuellement décrit, qui n'apparaît que si la production future est indépendante des dépenses publiques (par exemple déterminée par l'offre), ou si elle est anticipée être exogène,ce qui n'est naturellement pas le cas.

\section{QU'APPORTE LA «SOLVABILITÉ PRÉCOCE»?}

Normalement les agents économiques anticipent que le budget de l'Etat doit respecter la contrainte de solvabilité intertemporelle. Les contraintes incorporées dans le traité de Maastricht impliquent seulement une stabilisation relativement précoce du taux d'endettement public, qui n'est pas nécessaire pour assurer la solvabilité (celle-ci n'entraîne qu'une contrainte de long terme). Quelle est la logique derrière les critéres du traité du Maastricht ? A priori, ceux-ci sont sous-optimaux, puisqu'ils restreignent les politiques économiques qui ne devraient en principe n'imposer que la contrainte de solvabilité intertemporelle. Leur inclusion dans le traité peut résulter toutefois de différentes idées:

- les Etats ne s'imposaient pas la solvabilité intertemporelle;

- les anticipations des agents économiques sont naïves; observant que les dépenses publiques sont réduites rapidement, ils anticipent qu'elles continueront à l'être, ce qui stimule la consommation. En théorie, si aujourd'hui les dépenses publiques sont freinées, il y a au contraire davantage de marges de manoeuvre pour les accroître demain;

- les pays européens privilégient le bien-être de long terme qui est accru si le niveau initial de dette, dans quelques années, est plus faible. 


\section{PEUT-ON S'INSPIRER DES PETITS PAYS?}

Finalement, tous les pays européens vont devoir mener des politiques budgétaires restrictives, soit pour respecter les engagements liés à l'unification monétaire, soit simplement pour stabiliser leur taux d'endettement public.

L'exemple des petits pays montre qu'on peut le faire sans affecter la croissance, au contraire. 11 est clair que ce n'est pas l'accompagnement monétaire (baisse des taux d'intérêt, dévaluation...) qui, dans leur cas, a permis ce résultat. ll y a eu une brutale amélioration de la confiance des consommateurs, ce qui résulte sans doute d'un effet de seuil: au-delà d'un certain degré de détérioration des finances publiques, les consommateurs savent qu'il va y avoir une correction et, lorsqu'elle est entreprise, leurs anticipations s'améliorent. 11 n'est pas clair que la situation budgétaire des grands pays européens soit aujourd'hui suffisamment dégradée pour que ce même mécanisme stabilisateur joue.

\section{SUR LA NÉCESSITÉ DE RÈGLES BUDGÉTAIRES APRÈS L'UNIFICATION}

Les pays européens se sont mis d'accord sur des règles budgétaires assez strictes (le pacte de stabilité) après l'unification. Notre propos n'est pas ici d'examiner si ces règles ont été correctement conçues, par rapport, par exemple, à d'autres solutions plus coopératives, mais de nous demander s'il était vraiment nécessaire d'imposer une discipline budgétaire en Europe.

L'argument de base est celui des externalités nouvelles qui apparaissent après l'unification. Si un pays a un déficit excessif, en changes flexibles, lui seul en subit les conséquences (inflation, baisse des taux d'intérêt, dépréciation...). En union monétaire, les effets de ce déficit excessif se communiquent aux autres pays, par exemple par la hausse du taux d'intérêt commun. Il apparaît donc la nécessité nouvelle de contrôler les déficits excessifs, ce qui peut justifier le pacte de stabilité.

La réalité de ce type d'externalité a cependant été discutée.

Si un pays a un déficit excessif, et si les marchés financiers discriminent entre les émetteurs, seul le taux d'intérêt (en Euro) de ce pays augmente, c'est-à-dire qu'il subit un spread de taux vis-à-vis des meilleurs émetteurs en Euro. Dans ce cas, la discipline de marché joue, et les différents ont une forte incitation à ne pas accroître leur endettement.

Il n'est toutefois pas clair que la discrimination puisse effectivement jouer. Même si, en principe, aucun émetteur souverain défaillant ne sera secouru par les autres, en fait il est difficile d'imaginer que les pays européens puissent se désintéresser du sort d'un pays, émettant dans la monnaie commune, et en difficulté. S'il existe de fait une clause de sauvetage, alors cette solidarité implique bien que l'ensemble des taux d'intérêt européens serait affecté par la dégradation de la situation d'un des pays. 


\section{ZUSAMMENFASSUNG}

Wir analysieren die Glaubwürdigkeitswirkungen der Fiskalpolitik, einerseits auf der Ebene von angekündigten und tatsächlichen Defiziten und andererseits bezüglich der Konsequenzen einer restriktiven Politik. Es werden die Gründe untersucht, welche die Autoritäten dazu veranlassen können, solche Politiken anzukündigen und die Bedingungen unter denen sie glaubwürdig sind. Zudem werden auch die Wirkungen der Glaubwürdigkeit und der Restriktivität der Fiskalpolitik auf das Konsumverhalten, und damit auf die ökonomische Aktivität, betrachtet. Wir zeigen auf, welch bedeutende Rolle die Bestimmung und Erwartung der zukünftigen Produktion spielt.

\section{RÉSUMÉ}

Nous nous demandons ce qu'implique la crédibilité des politiques budgétaires, d'une part quant au niveau des déficits annoncés et réalisés, d'autre part quant aux effets des politiques de rigueur fiscale. Nous nous interrogeons sur les raisons qui poussent les autorités à annoncer de telles politiques, et sur les conditions pour qu'elles soient crédibles. Nous examinons en particulier les effets de la crédibilité et de la rigueur sur les comportements de consommation, donc sur l'activité économique. Nous montrons le rôle important joué par le mode de détermination et d'anticipation de la production future.

\section{SUMMARY}

We analyze the effects of credibility of fiscal policies, first on the levels of the announced and actual deficits, second on the consequences of restrictive fiscal policies. We examine the reasons for which the authorities can have an incentive to announce such policies, and the conditions under which those policies are credible. We also study the effects of credibility and fiscal restraint on consumption behavior; therefore on economic activity. We show the important role played by the way future production is determined and expected. 